\title{
Awkward Freedom. Freiheit und Programm bei Michael Snow
}

\section{Markus Klammer}

\section{Theatraler Formalismus}

Der folgende Text ist einer Reihe kinematographischer Arbeiten des 1929 geborenen kanadischen Künstlers Michael Snow gewidmet, einer der zentralen Figuren der Nachkriegsavantgarde. ${ }^{1}$ Es handelt sich um Filme, die zwischen Mitte der 1960er Jahre und Anfang der 1980er Jahre entstanden sind. Besonderes Augenmerk wird auf dem letzten Film dieser Reihe liegen, der 1981 veröffentlicht wurde und den Titel Presents trägt.

Snow verbrachte den prägenden Teil seiner Karriere in den 1960er Jahren in New York, nachdem er zuvor in Toronto als Zeichner an der Produktion von Animationsfilmen mitgewirkt und sich als Pianist im Rahmen von Jazzimprovisationen betätigt hatte. Den Jazz wird der Künstler bis in die radikalsten Spielarten des Free Jazz hinein sein Leben lang betreiben. Snow und seine Frau Joyce Wieland lebten von 1962 bis Anfang der 1970er Jahre in New York, bevor sie zurück nach Toronto zogen.

Das Werk des Kanadiers, den man immer noch vornehmlich als

experimentellen Filmemacher kennt, war seit den 1960er Jahren von außergewöhnlicher medialer Vielfalt, ja scheinbarer Heterogenität. Snow zeichnete, fertigte Gemälde, errichtete Skulpturen und Installationen, machte Filme, spielte Klavier, komponierte, fotografierte und vieles mehr. Diese Vielfalt an künstlerischen Praktiken und verwendeten Medien kommentierte der Künstler 1967 so:

I am not a professional. My paintings are done by a filmmaker sculpture by a musician, films by a painter, music by a filmmaker, 
paintings by a sculptor, sculpture by a filmmaker, films by a musician, music by a sculptor ... sometimes they all work together. ${ }^{2}$

In dem ebenfalls 1967 in der Juniausgabe der Zeitschrift Artforum erschienenen Aufsatz »Art and Objecthood« etablierte der Kunstkritiker und Kunsthistoriker Michael Fried eine grundlegende Differenz zwischen einer modernistischen Spielart der Künste, die dadurch charakterisiert ist, dass sie Werke innerhalb der Grenzen eines bestimmten Mediums schafft - etwa der Malerei oder der Skulptur -, und einem künstlerischen Modus, den Fried als »theatral« bezeichnete und für den er die Minimal Art als paradigmatisch ansah. Die Struktur theatraler Kunstwerke zeichnet sich Fried zufolge durch ihren forcierten, ja aufdringlichen Bezug zum Betrachter aus, wodurch dieser in ein zeitlich offenes Spiel von Wahrnehmungsvollzügen verwickelt wird, das Werk aber zugleich seiner Autonomie verlustig geht. In medialer Hinsicht wird Theatralität von Fried als das bestimmt, was zwischen den Künsten liegt: »The concepts of quality and value - and to the extent that these are central to art, the concept of art itself - are meaningful, or wholly meaningful, only within the individual arts. What lies between the arts is theater. ${ }^{3}$

Hält man die Aussagen von Snow und Fried, beide aus dem Jahr 1967, aneinander, so fällt es nicht schwer, Snow als einen Künstler zu erkennen, der die Theatralität - im sehr spezifischen Sinne Frieds, aber auch im landläufigen Sinne offensiv umarmt. Viele der Avantgardefilme Snows sind trotz ihres experimentellen Charakters voller theatraler Gesten und Mikrohandlungen, die ihre Wurzeln nicht selten im übertriebenen Schauspiel und im Slapstick des sogenannten `Stummfilms haben - von Wavelength (1967) über Standard Time (1967) und Back and Forth (1968-69) bis hin zu Rameau's Nephew by Diderot (Thanx to Dennis Young) by Wilma Schoen (1974), Presents (1981), To Lavoisier, Who Died in the Reign of Terror (1991) und zuletzt ${ }^{\star}$ Corpus Callosum (2001).

Die Theatralität Snows repräsentiert jedoch nur die eine Seite seines Filmemachens und seiner Kunst. Snow ist zugleich - und das macht ihn innerhalb der nordamerikanischen Avantgarde der 1960er und 1970er Jahre zu einer Figur von höchstem Interesse - erklärter und rigoroser Formalist, dessen Filme meist aus wenigen präzisen, vorher festgelegten Parametern generiert werden. Die eingangs zitierte Aussage Snows aus dem Jahr 1967 setzt sich folgendermaßen fort:

Also many of my paintings have been done by a painter, sculpture by a sculptor, films by a filmmaker, music by a musician. There is a tendency towards purity in all these media as separate endeavours. Painting as fixity, the static image. Sculpture's objectness. Light and Time. $\aleph^{4}$

Und noch 2009 antwortete der Künstler auf die Frage: »What is your approach to making art? «, mit dem Satz: "I'm a formalist. $\aleph^{5}$ Der Begriff `Formalismus« soll hier nicht nur in der von Snow aufgerufenen historischen Dimension verstanden werden - die Frontstellung Frieds gegen die Minimal Art als Auseinandersetzung um die rrichtige Auffassung des Formalismus in den Künsten -, sondern auch systematisch als Reduktion der jeweiligen sinnlichen Erscheinung eines Werks auf ihre Grundbedingungen bzw. als Transparentwerden der je spezifischen Erscheinungsweise eines Werks auf ihre Grundbedingungen hin.

\section{Ungelenke Freiheit}

Im Folgenden wird darzulegen sein, dass Theatralität und Formalismus - im Titel dieses Textes akzentuiert als »Freiheit« und »Programm « - in den Filmen Snows keinen Widerspruch darstellen. Vielmehr konstituieren sie die beiden Grundparameter von Snows Schaffen, aus deren Konfrontation sich nicht nur die spezifische Struktur seiner Filmwerke ergibt, sondern auch eine bestimmte Form ästhetischer Freiheit. Sie ist im Titel als »awkward freedom« bezeichnet, »ungelenke Freiheit«. »Awkward freedom», so die These, entsteht bei Snow gerade an der Schnittstelle von rigoroser, ja konzeptualistisch aufgefasster Form und dem, was man ausgehend von Fried »Theatralität« nennen kann.

In vielen Filmen Snows zeigt sich die Überkreuzung dieser beiden Parameter in Körperbewegungen. Unter >Körper sollen drei Dinge verstanden werden: A. Der Körper des Filmemachers, der seine - in der Regel - 16-mm-Kamera bedient. B. Die Körper von Personen, die in den projizierten Filmen auf der Ebene des Bildinhalts zu sehen sind. Auch die Körper von Tieren oder sich bewegenden unbelebten Gegenständen zählen zu dieser Gruppe. C. Schließlich der `Körper` des projizierten Filmbildes selbst. Damit sind dezidiert nicht die Kader des Filmstreifens aus Zelluloid gemeint, sondern das sichtbare, vom Kasch der Leinwand begrenzte Bildfeld des Films während der Projektion. Bei Snow wird die interne, visuelle Organisation dieses Bildfeldes mit seiner dem menschlichen Wahrnehmungsvollzug analogen Struktur mannigfachen Transformationen und Verzerrungen unterworfen. Dadurch wird es zuallererst in seiner `Körperlichkeit - man könnte auch sagen $>$ Medialität $<$ - erfahrbar.

Werden Körper - in einer der drei genannten Bedeutungen - bei Snow einem formalistischen Prinzip unterworfen oder versuchen sie, sich an bestimmte formalisierte Regeln oder Anweisungen zu halten, gelingt dies niemals mit absoluter Präzision. Im Scheitern der Körper an der selbst- oder fremdauferlegten Ausführung eines `Programms< entbindet sich bei Snow ein gestisches Moment, das zugleich ein genuin komisches Moment sein kann.

Der vorgeschlagene Begriff des $>$ Programms $\prec$ hat mehrere für Snows CEuvre maßgebliche Facetten. A. Erstens meint `Programm< ein Set von Handlungsvorschriften oder Regeln, die bei der Herstellung eines Werks leitend sind. In dieser Hinsicht besteht eine gewisse Verwandtschaft mit den von Allan Kaprow in Vorbereitung auf seine Happenings verteilten Programmen oder mit den meist in retrospektiver Vergangenheitsform verfassten Anweisungen, welche die fotografischen Elemente in Douglas Hueblers konzeptuellen Duration Pieces begleiten. Nicht zuletzt mag man an die zwischen Minimal Art und Konzeptkunst angesiedelten Partituren im Umfeld von Henry Flynt, Tony Conrad und La Monte Young denken, die den Charakter von Instruktion zur Ausführung bestimmter Handlungen trugen. ${ }^{6}$ 
Wie die Regeln eines Spiels haben jene Regeln Zwangscharakter. Ihre Befolgung lässt nur einen gewissen Spielraum. Auch wenn die Regeln nicht schriftlich niedergelegt oder in der fertigen Arbeit nicht unmittelbar als solche zu erkennen sind, müssen sie dennoch im Rahmen der Produktion des Werks explizit gemacht werden, um als Programm fungieren zu können. B. Zweitens besitzt >Programm< einen technischen Aspekt, wie beim Programmieren einer Maschine oder eines Computers. So verstanden bezeichnet der Ausdruck einen mehr oder weniger komplexen technischen Automatismus, dessen einfachste Form die wiederholte Ausführung eines bestimmten Ereignisses darstellt. Der technische Aspekt des Programms ist nicht auf Maschinen im engeren Sinne beschränkt. Auch der menschliche Körper kann als Maschine fungieren. C. Drittens hat $`$ Programm $`$ - das wurde bereits angedeutet - mit abstrahierter oder reduzierter Form zu tun. Der Technizismus des Programms in Snows Arbeiten ist auch ein Formalismus. ${ }^{7}$ Programme lassen sich als formale Strukturen verstehen, die ihrerseits mehr oder weniger komplexe Formen erzeugen. Bei Snow sind es dezidiert einfache Formen. D. Schließlich kann >Programm mäßige Prozesse meinen, die nicht mittels expliziter Programme bewerkstelligt wurden, die sich aber beschreiben lassen, als ob sie es wären, sei es im Sozialen, im Biologischen oder im Bereich der Physik.

Ein ähnliches Bedeutungsspektrum zeigt der vom Philosophen Stanley Cavell 1971 im Buch The World Viewed eingeführte Begriff des >Automatismus`, der noch ausführlich zu diskutieren sein wird. Cavell, in den 1960er Jahren ein enger intellektueller Weggefährte Frieds, unterscheidet zwei Typen von Automatismus hinsichtlich der Produktion von Kunstwerken. Der eine wird von den basalen technischen Eigenschaften eines Mediums vorgegeben, der andere bezeichnet den Gebrauch historisch sedimentierter ästhetischer Formen und Verfahren innerhalb einer Kunstgattung. ${ }^{8}$ In der modernistischen Kunst der 1960er Jahre sind beide Typen Cavell zufolge in eine Krise geraten und reichen nicht mehr hin, um überzeugende Werkformen zu begründen. Künstler wie Frank Stella oder Morris Louis stünden daher vor der Aufgabe, neue, die je spezifische Werkform tragende Automatismen einzuführen, »the task of establishing a new automatism«. ${ }^{9}$ Darüber hinaus heißt `Automatismus bei Cavell auch die Gesamtheit der von selbst nach Gesetzen ablaufenden Naturprozesse, "nature's autonomy «. ${ }^{10}$ Dass der vorliegende Text auf eine schlichte Übernahme der cavellschen Terminologie verzichtet, hat mehrere Gründe: Zum einen scheint der Begriff des `Programms der besonderen künstlerischen Praxis Snows - sein Umgang mit Körpern und Apparaten - angemessener, zum anderen ist er dialektisch auf seinen Gegenbegriff der `Freiheit‘ bezogen, eine Konstellation, die bei Cavell in dieser Weise nicht vorkommt, für eine detaillierte Interpretation von Snows Werk jedoch von großer Bedeutung ist.

Es versteht sich beinahe von selbst, dass mit dem Ausdruck >Freiheit $\prec$ keine «künstlerische Freiheit im juridischen Sinne oder im Sinne der schieren Unbeschränktheit kreativen Schaffens gemeint ist. Freiheit bei Snow bedeutet der hier vertretenen Auffassung nach eine Freiheit, die sich nur in Relation zu einem formalen Prinzip bzw. einem Programm zu etablieren vermag. Diese Freiheit nimmt häufig einen körperlich-gestischen Ausdruck an, der nicht im perfekten Gelingen einer geplanten Handlung oder einer Folge von Handlungen liegt, sondern in den Abweichungen und Verschiebungen bei deren Ausführung.

In der kantischen Philosophie wird die mit dem kategorischen Imperativ verbundene individuelle Freiheit definiert als eine Freiheit, von den eigenen Bedingtheiten als körperliches Wesen, von den eigenen Neigungen und sinnlichen Triebfedern abzusehen, um das Begehrungsvermögen durch ein rein formales Prinzip der Vernunft bestimmen zu lassen. Es ist eine Freiheit der Abstraktion, des Absehens von den empirischen, triebhaften Aspekten, die mit der menschlichen Existenz gleichwohl unauflöslich verbunden sind. ${ }^{11}$ Dem kantischen Formalismus des kategorischen Imperativs entspricht bei Snow der Formalismus des Programms. Die Körper in seinen Filmen aber können diesem Formalismus nicht gerecht werden. Die Unfälle und Zufälle des Misslingens bei Snow - häufig sind sie komisch und theatralisch zugleich - lassen eine linkische Form von Freiheit sichtbar werden.

Die kantische Freiheit beruht auf der Unabhängigkeit des Menschen als eines vernünftigen, »zur intelligiblen Welt gehörigen Wesens $\aleph^{12}$ von den Kausalzusammenhängen der Natur. Es ist eine Freiheit aus Autonomie. Die linkische Freiheit Snows hingegen stellt eine Freiheit aus Heteronomie dar. Ihre Voraussetzungen liegen gerade in der empirischen Welt, in den Bedingtheiten des Menschen als eines körperlichen Wesens, das mannigfachen Gesetzen und Regeln unterworfen ist.

\section{Körper - Apparat. Snows Filme und}

\section{ihre Verfahren}

Jene Filme, die im Spannungsfeld von Freiheit und Programm besonders interessieren, beschreibt der Künstler selbst als Werke, in denen es um die "presentation of camera movement almost for its own sake $\aleph^{13}$ gehe, also um die Thematisierung bestimmter Typen von Kamerabewegungen »beinahe um ihrer selbst willen«. Das Adverb `beinahe in dieser Aussage ist relevant, denn es verweist auf die Handlung als das Andere der filmischen Form. Die paradigmatischen Kamerabewegungen bei Snow sind der Zoom, der Schwenk in horizontaler und vertikaler Richtung sowie der sogenannte `Tracking Shot‘, die Kamerafahrt.

Snows wohl bekannteste filmische Arbeit ist Wavelength aus dem Jahr 1967. Der Film besteht aus einer 45-minütigen Zoombewegung, aufgenommen im New Yorker Atelier des Künstlers. Nachdem die Kamera optisch den ganzen Raum durchmessen hat, endet die von einem kontinuierlich ansteigenden Sinuston begleitete Zoombewegung auf einer formatfüllenden Fotografie des Meeres, die sich an der Wand zwischen zwei Fenstern an der Stirnseite des Raums befindet.

Das formale Programm des 45-minütigen Zooms wird von vier kurzen Szenen mit wechselndem Personal interpunktiert, welches im Kasch der Kamera wie auf einer Bühne agiert. Snow selbst spricht von »human events«, die mit dem sformal event der Zoombewegung kontrastieren. ${ }^{14}$ Die Handlungen sind banal und stereotypisiert. Ein Kasten wird gebracht. Zwei Frauen trinken Kaffee und hören den Song Strawberry Fields der Beatles. Ein Mann gerät ins Blickfeld der Kamera und mimt halbherzig seinen Tod. Schließlich kehrt eine der beiden Frauen 
zurück und fragt per Telefon um Rat, was sie mit dem Toten machen soll. Über all diese kleinen Geschichten gleitet der Zoom der Kamera hinweg.

Den drei vorgeschlagenen Semantiken von `Körper entsprechend, lässt sich das Konzept einer >ungelenken Freiheit an Wavelength exemplifizieren:

A. In Bezug auf den Körper des Filmemachers, der die Kamera führt: Der Zoom in Wavelength wird häufig als eine kontinuierliche, ununterbrochene Bewegung beschrieben. Das ist nicht ganz richtig. Einerseits ist der Film mehrfach geschnitten, wie unter anderem der plötzliche Wechsel von Tag und Nacht verdeutlicht, andererseits vollzieht sich - bei genauer Betrachtung - das Eindringen der Kamera in den Bildraum nicht kontinuierlich, sondern in kleinen Zoomschritten, die in nicht ganz regelmäßigen Abständen aufeinander folgen und einen optischen Rhythmus ergeben, der immer leicht aus dem Takt ist. Wir wissen von Snow selbst, dass er den Zoom der Kamera von Hand gezogen hat. In die scheinbare formale Unerbittlichkeit der Kamerabewegung schreibt sich so ein subtiler Gestus ein, der mit dem Körper des Künstlers, seiner Übung und seinen technischen Fertigkeiten, aber auch seiner Ermüdung und Unzulänglichkeit verbunden ist.

B. In Bezug auf die im projizierten filmischen Bild erscheinenden Personen: Das Verhältnis des menschlichen Körpers zur Kadrierung des filmischen, des fotografischen oder des gemalten Bildes stellt von Anfang an ein wichtiges künstlerisches Problem für Snow dar. Zwischen 1961 und 1967 suchte er in seinen Gemälden und Skulpturen, die sich um das Motiv der sogenannten Walking Woman entfalteten, nach einer Möglichkeit, die menschliche Figur mit Prinzipien formaler Abstraktion zu verbinden. Stellvertretend für andere Arbeiten der Serie können zwei skulpturale Versionen der Walking Woman aus dem frühen Film New York Eye and Ear Control (1964) herangezogen werden. Typisch sind die horizontalen Anschnitte von Kopf und Beinen, die auf die Kadrierung der Figur durch den - in der Skulptur freilich abwesenden - Bilderrahmen verweisen. ${ }^{15}$

In Wavelength hingegen scheint eine gewisse Unabhängigkeit, ja Gleichgültigkeit zwischen der Zoombewegung der Kamera und den Bewegungen und Handlungen der Figuren zu herrschen. Besonders deutlich wird dies bei dem auf dem Boden liegenden, unbeweglichen Körper, über den der Kasch der Kamera ungerührt hinwegzieht, nachdem sich der Protagonist - dargestellt vom Filmemacher Hollis Frampton - mehr oder weniger unmotiviert zum scheinbaren Sterben hingelegt hat. Nach den Konventionen des klassischen Erzählkinos stellt es eine Ungeschicklichkeit dar, wenn sich die Kamerabewegung allzu unabhängig von der Figurenhandlung vollzieht. Snow hingegen entkoppelt die beiden und setzt sie als jeweils für sich bestehende Elemente frei.

C. In Bezug auf die Körperlichkeit des Bildfeldes: Auf der Ebene des projizierten Bildes erzählt Wavelength die Geschichte der „Verflachung`, man könnte auch sagen der buchstäblichen >Bildwerdung s eines Raums - ebenfalls ein Grundthema in den Filmen Snows. Diese Verflachung vollzieht sich im vorliegenden Fall einerseits durch die optische Wirkung des Zooms, andererseits durch Doppelbelichtungen, die wie optische Filter funktionieren, und schließlich auf diegetischer Ebene durch den Zoom auf die Fotografie des Meeres mit ihrem prononciert flachen Bildträger.
Der 52-minütige Film Back and Forth entstand ein Jahr nach Wavelength. War bei Letzterem der Zoom das strukturierende formale Programm, ist es bei Back and Forth - wie bereits der Titel verrät - der Schwenk, und zwar in zunächst horizontaler, dann in vertikaler Ausrichtung. Das Setting ist ein Unterrichtsraum am Campus der Fairleigh Dickinson University in Madison, New Jersey. Die Bolex-Kamera schwenkt - auch hier von Snow selbst bedient - zunächst langsam von rechts nach links und von links nach rechts, um im Verlauf des Films immer schneller zu werden und schließlich in rasender Bewegung zu enden, die ein völliges Verschwimmen der auf dem Bild zu sehenden Gegenstände zur Folge hat, eine Verflachung des Raumes, die eine Abstraktion und Loslösung des filmischen Bildes von seinem Referenten bewirkt.

Die Schwenks der Kamera werden durch ein schlagendes Geräusch genau dann, wenn die Kamera kehrtmacht, akzentuiert, der visuelle Rhythmus so durch einen akustischen verdoppelt. Mit der Zeit hört und sieht man deutlich, dass die Intervalle und ihre Veränderungen dieselben feinen, gestischen Unregelmäßigkeiten aufweisen wie bei Wavelength, die an der Schnittstelle von Körper und Apparat entstehen. Für Snow existiert eine innige Verbindung zwischen der Filmkamera als einem optisch-technischen Aufnahmeinstrument und einem Musikinstrument wie dem Klavier: Beide registrieren die Körperbewegungen ihrer 'Spieler` und machen sie als akustische oder optische Phänomene erfahrbar.

Wie bei Wavelength wird auch in Back and Forth der Kasch des Filmbildes immer wieder von Menschengruppen bevölkert. Wir sehen sie in Lehroder Freizeitsituationen. Und wie im früheren Film vollziehen sich das Programm der Kamera und die kleinen, oft spielerischen menschlichen Handlungen und Interaktionen frei und unabhängig voneinander. Allerdings hat Snow auf diegetischer Ebene ein bemerkenswertes Motiv eingebaut, dasjenige eines Polizisten, der durch die Scheibe in den Seminarraum blickt. Der Polizist lässt sich als ironische Figuration des disziplinierenden Moments der repetitiven Kamerabewegung verstehen, aber auch als Anspielung auf die Auseinandersetzungen der amerikanischen Studentenbewegung mit der Staatsmacht in der zweiten Hälfte der 1960er Jahre.

Neben Wavelength ist La Région Centrale (1971) Snows bekanntester und zugleich am aufwendigsten produzierter Film. Er hat eine Dauer von etwas mehr als drei Stunden. Auch hier setzt sich das in Wavelength und Back and Forth, aber auch in Standard Time erprobte Thema der Kamerabewegungen fort. Der Film wurde auf einem Hochplateau in Kanada in der Provinz Québec nördlich der Stadt Sept-Îles gedreht. Der Schauplatz verlagert sich im Vergleich mit den früheren kinematographischen Arbeiten von Zimmern und Innenräumen in die freie Natur. Dem Künstler zufolge wohnt dem Film eine »kosmologische« Dimension inne: Zeitlich gerafft stellt er den Ablauf eines 24-stündigen Tages dar. ${ }^{16}$

Für La Région Centrale ließ Snow einen aufwendigen Apparat bauen, den man mittels Hubschrauber auf das entlegene Hochplateau transportierte und dort fest im Boden verankerte. Er glich einem hypertrophen Karussell und konnte vier `Freiheiten der Kamerabewegung - separat und miteinander kombiniert - ausführen: Zoom, Schwenk horizontal, Schwenk vertikal und Drehung der Kamera 
um die eigene Achse. Die im Lauf des Films variierte Grundbewegung der Kamera bildet ein einfacher horizontaler Schwenk um 360 Grad, also eine Kreisbewegung. Die ausgeklügelte technische Apparatur ist im Bildfeld des projizierten Films niemals direkt sichtbar, nur ihr Schlagschatten am steinigen Boden gerät hin und wieder ins Bild, wobei die Sonne als eine Art gigantischer natürlicher Projektor fungiert.

Das Thema der Verflachung und Abstraktion des filmischen Bildes durch Bewegungsunschärfen - und damit seiner Befreiung vom Referenten - spielt auch in La Région Centrale eine wesentliche Rolle, wie sich aus den wirbelnden, rasend-crescendierenden Rotationsbewegungen der Kamera am Ende des Werks ersehen lässt. Im Vergleich mit den bisher vorgestellten Filmen Snows scheinen der Körper des Filmemachers und das mit ihm verbundene gestische Potenzial jedoch von keinerlei Bedeutung zu sein. Vielmehr scheint sich die filmische Bewegung einer vorprogrammierten mechanischen Apparatur zu verdanken, welche die Bildproduktion von ihrer Gebundenheit an den trägen menschlichen Körper und seine Struktur befreit, die nach den Parametern von oben und unten, vorne und hinten, links und rechts differenziert. ${ }^{17}$

Bekanntlich hat Gilles Deleuze in seinen Kinobüchern die Bilder von La Région Centrale als Paradigmen reiner optischer Perzepte verstanden, die ihrer Form nach nicht mehr an einen wahrnehmenden menschlichen Leib gebunden sind. ${ }^{18}$ Folgt man Deleuze, so ist mit La Région Centrale möglicherweise ein Gegenbeispiel zum hier behaupteten Grundzug einer sungelenken Freiheit im Experimentalfilmschaffen Snows gefunden. Denn erstens fehlen auf der Seite des Bildinhalts Figuren und theatralische mikrodramatische Szenen völlig, und zweitens scheint die Kamerabewegung dem unerbittlichen, ahumanen Programm einer komplexen Maschine zu folgen. ${ }^{19}$

Das erste dieser Argumente muss zugestanden werden, das zweite jedoch erweist sich als nicht haltbar, im Gegenteil. Snow steuerte die Kamera nämlich - hinter einem Felsen verborgen - mittels eines im Boden verlegten Kabels fern. In vielen seiner publizierten Interviews vergleicht der Künstler den Verbund von Fernbedienung und Kameraapparatur mit einem Musikinstrument, das er vor Ort in der Wildnis des Hochplateaus gleichsam sspielter. Tatsächlich hatte Snow vor den Dreharbeiten eine Partitur der Kamerabewegungen und ihrer Kombinationen angefertigt, von der er aber im Verlauf der Filmaufnahmen - aus unterschiedlichen Gründen - immer wieder abwich und `frei improvisierter. Ursprünglich hatte er sogar geplant, die Partitur mittels eines Synthesizers auf Tonband aufzunehmen, um die Kameramaschine dann mit dem Tonband wie mit einem Computerprogramm zu steuern. Dabei wären den vier Grundbewegungen der Kamera vier sinusähnliche Töne unterschiedlicher Höhe zugewiesen worden, welche im Apparat die entsprechende Kameraaktion ausgelöst hätten. Snow und seinem Ingenieur Pierre Abbeloos wurde jedoch bald klar, dass die Verwirklichung dieses Vorhabens zwar technisch realisierbar, in der Praxis aber zu aufwendig war. ${ }^{20}$

In den fertigen Film hat der ursprüngliche Plan dennoch Eingang gefunden. Snow spielte die den Grundbewegungen der Kamera entsprechenden Töne nachträglich im Studio mit einem Synthesizer ein, während er den bereits geschnittenen Film im Projektor laufen ließ. Aus diesem Verfahren aber ergaben sich charakteristische Abweichungen im endgültigen Werk. Die Töne und die Veränderungen im Bewegungsschema der Kamera sind nicht exakt synchronisiert, manchmal geht der Ton einer Bewegungsänderung voraus, manchmal folgt er, manchmal fällt er genau mit ihr zusammen.

Diese leichten Abweichungen nun schreiben das `Spiek des Künstlers auf den diversen an der Produktion von La Région Centrale beteiligten technischen Instrumenten in die Projektion des Films selbst ein. Sie erzeugen eine Mise en abyme von Kausalitäten, ein Verwirrspiel von Ursache und Wirkung, da sich während der Betrachtung des Films keine stabilen Hypothesen darüber bilden lassen, ob die Töne nun als `Befehle< fungieren, welche Bildwirkungen auslösen, oder ob umgekehrt die Bilder bestimmte Töne hervorbringen. In dieser minimalen Unbestimmtheitszone siedelt die linkische und doch so elaborierte Freiheit von La Région Centrale.

\section{Presents}

Alle namhaft gemachten Komponenten der linkischen Freiheit Snows finden sich zusammengefasst und gesteigert im Film Presents. Besonders der theatrale, komödienhafte Aspekt von Freiheit im Cuvre des Künstlers kommt hier zu seinem vollen Recht. Darauf deutet schon der Titel hin, den man »Presents« - im Deutschen also »Geschenke« oder auch »Gegenwart«, »presence« - oder »Presents« aussprechen kann, wobei in letzterem Fall die 3. Person Singular des Verbs »to present«, »vorstellen, präsentieren« ausgedrückt wird: »er/sie/es präsentiert«, wie ein Impresario in einem Theater oder einem Zirkus. Snow hatte den Film nach einer siebenjährigen Pause als erste größere Arbeit nach seinem rund viereinhalbstündigen Werk Rameau's Nephew by Diderot von 1974 fertiggestellt.

Der Film Presents weist, wie bereits erwähnt, direkte Bezüge zum Dispositiv des Theaters auf und besteht aus drei unterschiedlich langen >Akten Unverkennbar ist eine Auseinandersetzung mit dem einflussreichen, von Fried in seinen historischen Ramifikationen untersuchten absorptiven Theatermodell von Denis Diderot, dem die »ontologische Fiktion ${ }^{21}$ einer absoluten Trennung zwischen der ästhetischen Welt des Theaterstücks und der empirischen Welt des Zuschauers zugrunde liegt. Stärker jedoch dominiert ein Theatermodell, das Fried als »theatral« im engeren Sinne bezeichnet. ${ }^{22}$ In Letzterem wird das Publikum frontal adressiert, seine Gegenwart wird unmittelbar anerkannt und für eine Reihe von Tricks und Effekten genützt. Man könnte es auch als `kommunikatives` oder `spektakuläres` Theatermodell bezeichnen.

Der kurze erste Akt zeigt die von einem sich langsam verändernden sinusähnlichen Ton begleitete elektronische Transformation des Videobildes einer schlafenden, unbekleideten Frau, die nach Art von Francisco de Goyas Nackter Maya - manche Interpreten fühlen sich auch an Edouard Manets Olympia erinnert in einem kulissenhaften Innenraum liegt. ${ }^{23}$ Der erste Akt nimmt die Thematik der Transformationen und Verzerrungen des Bildfeldes und der in ihm enthaltenen Objekte wieder auf. Sie erhält in Presents eine medienspezifische Wendung, indem sie explizit an die Technik des Videos geknüpft ist - und nicht an den Zelluloidfilm, 
mit dem sich die angestrebten Effekte nicht erreichen ließen. Zudem zeigt der erste Akt mit der schlafenden, sich wie auf einem Gemälde kaum bewegenden Frau wesentliche Merkmale absorptiver Bildlichkeit nach Fried, während der zweite Akt eindeutig theatral ist und der dritte Akt eine Synthese zwischen der Absorption des ersten Aktes und der Theatralität des zweiten Aktes darstellt.

Der zweite Akt ist, ebenso wie der dritte, auf 16-mm-Material gefilmt. Er spielt auf einer merkwürdig kulissenhaften, improvisiert und instabil wirkenden Theaterbühne. Die Frau aus dem ersten Akt verlässt auf einen einzelnen Trommelschlag hin ihr Bettlager, streift sich einen mondänen Morgenmantel über und empfängt in ihrem Wohnzimmer einen linkischen Mann, der ihr Blumen schenkt. Die Kamera folgt ihren Handlungen augenscheinlich mittels strikt lateraler Bewegungen. Dann suchen die beiden einen Gegenstand, ein Bild, das sie schließlich finden. Auf der Tonebene wird der zweite Akt von Bachs Solosuiten für Violoncello begleitet, die von einem Plattenspieler kommen, der sich direkt auf der Bühne befindet und von der weiblichen Protagonistin in Gang gesetzt wurde.

Es ist unschwer festzustellen, dass es auch in diesem Fall um besondere Kamerabewegungen geht, nämlich um die horizontale, streng bildparallele Kamerafahrt und den Zoom. Wodurch aber entstehen die seltsamen Verrenkungen der Schauspieler, wodurch entsteht das Ruckeln und Wackeln des Dekors sowie das wiederholte irritierende Springen der Nadel auf dem Plattenteller? Die Antwort lautet: In Presents bewegt Snow nicht die Kamera, vielmehr bewegt sich die gesamte Bühne mit Schauspielern und Dekor: Sie wird von einem riesigen, nicht sicht-, aber hörbaren Gabelstapler vor einer völlig statischen Kamera hin- und her-, vor- und zurückgeschoben. Aus dem Off gebrüllte Kommandos des Filmemachers begleiten die einzelnen Operationen des Gabelstaplers: »One!«, »Two!«, »Three!«, »Four!« und so fort bis "Nine!«. Wie in La Région Centrale bleibt auch hier das kausale Verhältnis zwischen akustischem Kommando und maschineller Aktion im Unbestimmten. Löst das Kommando die Aktion aus, begleitet es sie oder bildet es bloß ihren Nachhall und Kommentar? In jedem Fall gehört die Verschränkung der musikalischen Rhythmen von Bachs erster Cellosuite, der gezählten Zeit der mündlichen Kommandos und der ratternden, brummenden, aufheulenden Motorgeräusche des Gabelstaplers auf formaler Ebene zum Witzigsten und Optimistischsten, was der Avantgardefilm der zweiten Hälfte des 20. Jahrhunderts hervorgebracht hat.

Der anarchische letzte Teil des zweiten Aktes schließlich, in welchem die Bühnenausstattung zu zerfallen und zu implodieren scheint, wurde mit einem von Snow selbst pilotierten, an einen hypertrophen Rollstuhl erinnernden Kamerawagen aufgenommen, vor den eine durchsichtige, gleichwohl massive Plexiglasplatte montiert war. Der Wagen bewegte sich wie ein Schneepflug schwerfällig und ruckartig über die Bühne und zerstörte, was ihm in den Weg kam. Im projizierten Film hingegen sieht es so aus, als wären die Gegenstände durch ihre schiere visuelle Präsenz im Kasch einem Feld unsichtbarer Kräfte unterworfen, das sie mehr oder weniger abrupt kollabieren lässt.

Der zweite Akt von Presents ist durch eine forcierte Theatralität gekennzeichnet, der bei aller Komik und Lächerlichkeit ein selbstreflexiver Zug innewohnt: A. Die Schäbigkeit und Behelfsmäßigkeit des Bühnenraums legt dessen konstruierten Charakter bloß. B. Der Motorlärm des Gabelstaplers und die Kommandos aus dem Off verweisen auf die Produktionsseite des Films, auf die Programme, die seine Erscheinungsweise bedingen. C. Auf der transparenten Plexiglasplatte des Kamerarollstuhls sind - zumal vor dunklem Hintergrund - Spiegelungen zu erkennen, welche schemenhaft den lenkenden, mit einer schwarzen Balaklava bekleideten Filmemacher und die oberhalb der Lehne befestigte Kamera sichtbar machen oder hektische Aufräumbewegungen von Bühnengehilfen im Rücken des Gefährts erscheinen lassen. D. Der zweite Akt gliedert sich seinerseits in drei >Aufzüge`, die von zwei kurzen Pausen unterbrochen werden. Die erste Pause besteht aus einigen Sekunden Schwarzfilm. Sie wird von einer langsamen Drehbewegung des Bühnenraums aus der Bildparallele nach hinten eingeleitet - das Metallgerüst an der Unterseite der Bühne kommt auf diese Weise zum Vorschein - und durch die inverse Bewegung von schräg hinten nach vorne in die Bildparallele beendet. Unmittelbar vor der zweiten Pause ist das von den handelnden Figuren gesuchte Bild endlich gefunden. Die Frau reicht es dem Mann und tritt dann nach vorne an den Bühnenrand, sodass Kopf und Füße vom Kasch abgeschnitten werden. Sie macht sich oberhalb des Bildrandes zu schaffen. Ein Bühnenvorhang schiebt sich gleichzeitig von rechts und links vor die Szene und färbt das Bildfeld schwarz, bis auf einen schmalen vertikalen Schlitz, der einen minimalen, beinahe abstrakten Durchblick auf die rote Rückwand und den blauen Boden gewährt. Es folgt eine kurze Strecke Schwarzfilms. Dann ist der Schlitz wieder da, und die Protagonistin bewegt die beiden Vorhangteile zur Seite, so wie sie diese zuvor geschlossen hat. Die unterschiedliche Struktur der beiden Pausen macht es leicht, die erste Pause ihrer Form nach dem Film - sie wird von einer Ab- und einer Aufblende gerahmt und die zweite dem Theater zuzuschreiben - sie wird vom Zu- und Aufziehen eines Vorhangs umschlossen. Insbesondere die zweite Pause bringt die Strategie, das Bildfeld des Films mit einem Theaterraum gleichzusetzen, auf den Punkt. E. Die banale Handlung und das übertriebene Schauspiel stellen ihre eigene Künstlichkeit aus. Die ruckartigen Bewegungen der Bühne zwingen die Schauspieler aus ihren gewohnten motorischen Mustern und erlegen ihnen neue Herausforderungen auf, zum Beispiel ১Haltung zu bewahren, obwohl man dabei ist, das Gleichgewicht zu verlieren, oder nicht zu schnell und nicht zu langsam am Stand zu gehen, um buchstäblich nicht saus dem Bild zu fallen،. Dadurch werden slapstickhafte Bewegungsabläufe generiert, die zugleich unbeholfen und virtuos wirken. ${ }^{24}$

Der dritte und letzte Akt des Films besteht aus rund zweitausend Einstellungen - genauer gesagt Kameraschwenks -, die buchstäblich nur einen Augenblick dauern. Die sehr mobile Handkamera Snows fungiert als Äquivalent zu einem befreiten Blick, dessen Aufmerksamkeit plötzlich durch die Bewegung eines Gegenstandes angezogen wird, dem er spielerisch folgt, um sich dann wieder abzuwenden. Die Myriaden Aufnahmen des dritten Aktes weisen alle unterschiedliche Längen auf. Die optischen Schnitte werden jeweils durch Trommelschläge akustisch verdoppelt, deren Folge sich ganz am Ende des Films zu einem prasselnden Trommelwirbel steigert. Die Aufnahmen sind lose aufeinander bezogen, ihre Montage folgt 
keinem strikten Programm, sondern einer Art freischwebender filmischer Aufmerksamkeit. Die Kamera lässt sich von den kontingenten Bewegungen der Welt ihr Maß geben.

Bestimmte Bewegungen der gezeigten Gegenstände bzw. der Kamera, die ihnen folgt, setzen sich über mehrere Einstellungen fort oder sie antworten dialogisch aufeinander, etwa wenn ein vertikaler Schwenk nach einem horizontalen kommt. Thema all dieser Filmbilder ist die freie Bewegung im visuellen Feld der Kamera: Der dritte Akt von Presents zeigt Bewegungen von Tieren und Pflanzen ebenso wie von Verkehrsmitteln und Maschinen, er verbindet die Jahreszeiten, die Klimazonen und die Elemente. Leitmotivisch taucht - neben Hubschraubern und Flugzeugen - immer wieder der Flug von Vögeln, alleine oder im Schwarm, auf.

Das Kurze, Blickhafte der filmischen Schwenks verweist nach Snows eigener Aussage auf eine der möglichen Bedeutungen des Filmtitels, nämlich »Gegenwart « - »Presents« als "presence«, »Gegenwart«. Die Aufnahmen des dritten Aktes entsprechen flüchtigen Wahrnehmungseindrücken, die kurz gegenwärtig sind, um von neuen Eindrücken abgelöst zu werden, die ebenso schnell wieder in der Vergangenheit versinken. Mit der fortwährenden Dauer der Filmbetrachtung aber bilden sich formale und inhaltliche Korrespondenzen zwischen den Myriaden von kurzen filmischen Gegenwarten. ${ }^{25}$

War die scheinbar mechanische Bewegung der Kamera in den Filmen vor Presents unmittelbar von einem gestischen Ausdruck durchzogen, welcher von den mehr oder weniger präzisen Bewegungen des Körpers des Filmemachers herrührte, sind diese beiden Elemente im Film von 1981 getrennt und auf den zweiten und dritten Akt verteilt: Der zweite Akt führt die mechanische Dimension der Kamerabewegung auf geradezu groteske Weise vor Augen, während der dritte Akt die Flüchtigkeit des Blickens mit der spielerischen Leichtigkeit von Hand- und Armbewegungen des Filmenden verknüpft. Während die Schauspieler im zweiten Akt auf virtuose Weise gegen die ruckenden Bewegungen der Bühne um ein kleines Stück rawkward freedom kämpfen und dabei neue Bewegungsmuster erfinden müssen, um nicht zu fallen oder aus dem Bildfeld zu geraten, sind die Bilder des dritten Aktes von einer großen Leichtigkeit, und sie versöhnen unser Schicksal als Menschen körperliche, schwere Wesen zu sein - mit der freien Ungebundenheit des Blickens. ${ }^{26}$

\section{Ernste Komödie}

In seinen Untersuchungen von Diderots Theaterkonzeption hat der Komparatist Peter Szondi auf eine Isomorphie zwischen der dramatischen Form und den dargestellten Stoffen bei Diderot aufmerksam gemacht: Dem Postulat einer radikalen ästhetischen Trennung der Welt der Bühnenhandlung von der Welt des Publikums, ihrer Versiegelung und Stillstellung in einem Tableau, welches sich gleichwohl mit Inbrunst und Rührung betrachten lässt, entspreche der von Diderot affirmierte Kult der »Privatisierung des Lebens $\ll^{27}$ der sich im 18. Jahrhundert neu formierenden bürgerlichen Kleinfamilie, ihr Bedürfnis nach Abschottung gegen die Welt des Adels und seine Intrigen, schließlich eine neue Form der »dauerhaften Intimität des [...] Familienlebens $\ll^{28}$ und der mit dieser verbundenen empfindsamen
Regungen und zärtlichen Affekte. Zugleich versäumt es Szondi nicht, eine politische Dimension des diderotschen Absorptionsparadigmas zu benennen: Diderot, so Szondi, gebrauch

»die tragédie domestique et bourgeoise zur Darstellung und Verklärung der bürgerlichen Kleinfamilie als realer Utopie, in deren Abgeschiedenheit der rechtlose Bürger seine Ohnmacht in der absoluten Monarchie vergessen und sich entgegen allem Augenschein der Güte der menschlichen Natur versichern kann ${ }^{29}$

Es sind zwei von Diderot propagierte Theatergattungen, welche die immanente Güte der menschlichen Natur am besten zur Geltung bringen: das bürgerliche Trauerspiel und die ernste Komödie, die Diderot im "genre sérieux" zusammenfasst. ${ }^{30}$ In der ernsten Komödie steht nicht das Lachen über die Bosheiten und Schwächen der Menschen im Vordergrund, sondern die mit der Erfüllung menschlicher Pflichten einhergehende Süße und Heiterkeit.

Der zweite Akt von Presents nun lässt sich geradezu als Umkehrung der ernsten Komödie Diderots verstehen. ${ }^{31}$ Das seltsame Paar im billigen, generischen Interieur der frühen 1980er Jahre kann als historische Schwund- und Endstufe der bürgerlichen Familie des 18. Jahrhunderts angesehen werden. Durch die Ungeschicklichkeit der agierenden Personen scheint die Etablierung einer Familie trotz des Blumengeschenks des Mannes - schon im Stadium der Anbahnung zu scheitern. Noch lange, nachdem er eingetreten ist, trägt der Mann seine dicken, orangen Winterhandschuhe. Die Mütze wird er gar nicht abnehmen. Die Umgangsformen, welche die beiden Figuren bei Snow miteinander pflegen, sind keine zärtlichen, intimen, vertrauten. Vielmehr scheinen sie überhaupt keine Innerlichkeit $\mathrm{zu}$ besitzen, keine Empfindungen, Regungen und Affekte, sondern ein Repertoire von sozialen Formen, sprachlichen Floskeln und körperlichen Bewegungsmustern abzuspulen. Dementsprechend entsteht das komische Element nicht, wie bei Diderot vorgesehen, durch die Heiterkeit, welche die Einsicht in die eigenen Pflichten gewährt, sondern durch das Scheitern der beiden Figuren an der einfachsten Mechanik der sozialen und sprachlichen Formen, an den simpelsten Abfolgen banaler Körperbewegungen. Schließlich steht Diderots Ästhetik der Absorption eine forcierte Theatralität bei Snow gegenüber.

Und dennoch ergeben sich bemerkenswerte Parallelen: Bei Snow ebenso wie bei Diderot verweist das Komische auf die conditio humana in ihrer anthropologischen und in ihrer spezifisch historischen Dimension, und bei Snow ebenso wie bei Diderot fungiert dieser Verweis als ästhetische Ermöglichungsbedingung von Freiheit. Was sich geändert hat, ist der Referenzrahmen der conditio humana. Bei Diderot ist es ein idealistischer, bei Snow wird er von Programmen im weitesten Sinne gebildet - ob sie nun technischer, ästhetischer, biologischer oder sozialer Art sind.

Die vielen kurzen, durch Kameraschwenks bewegten Einstellungen des dritten Aktes filmte Snow - vermutlich während mehrerer Jahre - zu Hause und im Zuge von Reisen auf unterschiedlichen Kontinenten. Sie erinnern 
an Versatzstücke von Home Movies und Urlaubsfilmen, wie sie auch ein ambitionierter Super-8-Filmamateur hätte drehen können. Der Künstler selbst betont ihren privaten Charakter. ${ }^{32}$ Wie im vorangegangenen Abschnitt dargelegt, sublimieren sie in ihrer losen Ordnung als freie filmische Blicke ohne eigentlichen Blickträger die mühevolle Arbeit der Koordination körperlicher Bewegungsabläufe, sozialer Formen und des kinematographischen Produktionsdispositivs, welche dem zweiten Akt von Presents seine komödienhaften Züge gibt. Zugleich unterstreicht Snow den »tragischen « Aspekt der schieren Flüchtigkeit der Bilder. ${ }^{33}$ Der dritte Akt verleiht dem Werk einen Ernst, der nicht zuletzt von der kleinbürgerlichen Praxis des Sammelns, Aufbewahrens und Vorzeigens vergänglicher privater Momente im Medium des Films getragen ist. Er transformiert diese Praxis in die Sprache der Avantgarde und versetzt das Werk in den Stand einer ernsten Komödie des späten 20. Jahrhunderts.

Dabei muss beachtet werden, dass die komödiantischen Elemente des zweiten Aktes durchwegs an die Tragik des Scheiterns an und von Programmen geknüpft sind. Umgekehrt finden sich die Gewalt - man denke an die Zerstörung des Interieurs - und das Triebhafte - der von Snow pilotierte Kamerawagen rammt wiederholt das Gesäß der Protagonistin, sein Blickfeld gemahnt an ein lechzendes Tier,$-{ }^{34}$ die im zweiten Akt thematisch sind, spurhaft im dritten Akt wieder: in Gestalt von Operationsszenen, von Bildern, welche die Jagd und Zerteilung eines Rentiers im Schnee zeigen, oder von Aufnahmen unbekleideter Frauen in Innenräumen. Die freie Form - und das ist charakteristisch für Snows CEuvre als Ganzes ist durchzogen von den ıniederen« Regungen der Triebe und des Begehrens.

\section{Modernistische "seriousness"}

Man kann sich dem Problem einer avantgardistischen Neubegründung der ernsten Komödie bei Snow aber noch auf einem anderen Weg nähern, nämlich über Cavell, in dessen Bestimmung des Modernismus die Kategorien des Ernstes und des Komischen eine zentrale Rolle spielen. Im Folgenden soll deshalb kurz auf das Gesamtprojekt von The World Viewed und seine Verflechtungen mit Frieds Kunsttheorie eingegangen werden.

Die Moderne ist für Cavell durch eine unheilvolle epistemologische Trennung von Subjekt und Welt charakterisiert, die der Philosoph ausgehend von Martin Heideggers Analyse des Cartesianismus in "Die Zeit des Weltbildes« begreift. $^{35}$ Sie drückt sich unter anderem aus als »our knowledge that we are lost to nature, are absent from it, cannot face it ${ }^{36}$ Die modernistische Kunst sucht diese Trennung zu heilen, eine Gemeinschaft zwischen Subjekt und Welt und zwischen den menschlichen Individuen untereinander herzustellen. Aber sie kann die Trennung nicht einfach überwinden. Was der Kunst bleibt, ist, die historische Situation der Moderne in der spezifischen Form ihrer Werke maximal zuzuspitzen, Cavell sagt in Anlehnung an Fried »anzuerkennen«. Diese Anerkennung macht den ontologischen Bruch von Selbst und Welt nicht ungeschehen, doch hebt sie ihn auf im hegelschen Sinne, indem sie ihm ästhetische Form verleiht.

Die Struktur dieser Form trägt bei Cavell den Namen »Autonomie«, bei Fried heißt sie »Antitheatralität« und ab Mitte der 1970er Jahre »Absorption«.
Das autonome Werk ist für Cavell ein Werk, dessen Erscheinungsweise nicht durch die Automatismen traditioneller Gattungen und überlieferter Formbestände geregelt ist. Es muss seine ästhetischen Verfahren und seine künstlerische Überzeugungskraft zuallererst durch sich selbst legitimieren. Durch seine selbstreferenzielle SchlieBung wird das gelungene modernistische Werk zu einer 'Welt‘ oder 'Natur zweiter Ordnung und spiegelt so die Trennung von empirischer Welt und erkennendem Subjekt. Zugleich aber steht das modernistische Werk Cavell zufolge für das Subjekt in der Moderne selbst, denn wie Letzteres ist es in ständiger Gefahr zu scheitern: Wie dem modernen Subjekt sind ihm seine Formen nicht rautomatisch vorgegeben, es ist bedroht von Kontingenz. Dem modernistischen Werk eignet daher eine zutiefst ethische Dimension: Die gelungene Werkform gibt dem betrachtenden Subjekt die berechtigte Hoffnung, auch sein Leben werde schließlich eine gelingende Form finden. ${ }^{37}$ Der Vorschein jenes Gelingens ist die Konfrontation mit dem modernistischen Werk: »In response to modern painting, I am concentrated, finitized, incarnate. $\aleph^{38}$

Wie nun begreift Cavell die gelingende autonome Form des modernistischen Werks? Eine derartige Form, so Cavell, muss aus der Erfindung neuer Automatismen hervorgehen. ${ }^{39}$ Wie bereits ausgeführt, kennt Cavell zwei Arten von Automatismus in den Künsten, einen Automatismus, der auf den grundlegenden technisch-medialen Eigenschaften der Darstellungsmittel einer Kunstgattung beruht, und einen Automatismus, der sich aus dem innerhalb einer Kunstgattung überlieferten Bestand an ästhetischen Formen und Verfahren sowie den Regeln ihrer Anwendung speist. In der Moderne haben die ästhetischen Automatismen in den einzelnen Künsten, Cavells und Frieds Diagnose zufolge, ihre normative Kraft verloren, zugleich sind die von der jeweiligen technisch-medialen Basis der Künste vorgegebenen Automatismen - man denke an Clement Greenbergs Paradigma der »flatness « modernistischer Malerei - zu einfach, um überzeugende Kunstwerke ergeben zu können. ${ }^{40}$ Erst die experimentelle Erschließung neuer Automatismen, die trotz ihrer radikalen Neuheit einen reflexiven Bezug zu den technisch-medialen und historischen Voraussetzungen einer bestimmten Kunst wie der Malerei oder der Musik bewahren, vermag Abhilfe zu schaffen. ${ }^{41}$

Fungierte in Cavells erstmals 1967 veröffentlichtem Aufsatz »Music Discomposed « die Musik als paradigmatische modernistische Kunst, ${ }^{42}$ ist es vier Jahre später in The World Viewed - nicht zuletzt aufgrund der mit Fried geführten Diskussionen - die Malerei. In enger Anlehnung an Frieds epochemachenden Essay »Art and Objecthood« beschreibt Cavell die Qualität eines gelungenen modernistischen Gemäldes als "total thereness «, "presentness «, »absolute hereness and nowness" »candidness «, »completeness « ${ }^{43}$ aber auch als $»$ haecceity $«{ }^{44}$ Diese Prädikate bezeich nen nichts anderes als die in sich geschlossene Form des modernistischen Werks, welche nur aus sich selbst heraus, ohne den Rückgriff auf etablierte Automatismen technischer oder ästhetischer Art, zu überzeugen vermag. Das gelungene modernistische Werk bildet kraft seiner Form eine in sich geschlossene, radikal von der Sphäre des Betrachters getrennte Welt. Cavell spricht von einem »Rückzug« der Welt, der nicht nur die eigene »presentness«, die eigene ereignishafte (Selbst-) Gegenwärtigkeit des Werks, anerkenne, sondern dialektisch auch die der betrachtenden Subjekte. ${ }^{45}$ 
Damit stellt der Philosoph die modernistische Malerei implizit in die Tradition Diderots und seiner Bestimmung des `Tableaus in den Künsten des 18. Jahrhunderts. Es ist diese Genealogie, die Fried wenige Jahre später mit Absorption and Theatricality und seither in fast jeder seiner Studien ausdrücklich zum Thema machen wird. Tableau zu sein, heißt für ein Gemälde oder ein Theaterstück des 18. Jahrhunderts, sich von der Welt des Publikums abzuschließen, betrachtbar zu werden als ein für sich seiendes Ganzes - nicht allein der Form nach wie in der modernistischen Malerei der 1960er Jahre, sondern auch den Stoffen und Motiven nach: Man bevorzugte die Darstellung bestimmter selbstversunkener, intimer, häuslicher Tätigkeiten. Theatralität als Gegenbegriff zur Absorption bedeutet in diesem Paradigma, die Trennung zwischen Werk und Betrachter nicht zu respektieren, direkt durch Reize, Signale und Effekte an den Betrachter zu appellieren, um seine Aufmerksamkeit zu heischen, eine falsche Kontinuität zwischen der ästhetischen Sphäre und der empirischen Welt zu suggerieren.

Keine der Gattungen im klassischen Kanon der Künste ist von Haus aus absorptiv und antitheatral. Erst der spezifische Gebrauch der jeweils zur Verfügung stehenden künstlerischen Mittel vermag eine Ästhetik der Absorption und Antitheatralität zu begründen. Anders verhält es sich im Fall des Films, wie sowohl Fried als auch Cavell betonen. Dem Film sei seine Antitheatralität bereits qua Medium inhärent. Seine technisch-mediale Basis garantiert die radikale Trennung zwischen der - auch in der Gegenwart der Projektion - strukturell vergangenen Welt des Films und der Welt des Publikums. ${ }^{46}$ Die Autonomie des Films liegt für Cavell in der Weise seiner Herstellung und seiner Projektion begründet. Beide erfolgen automatisch, »removing the human agent from the task of reproduction $\ll .{ }^{47}$ An dieser Stelle zeigt sich eine weitere, für den Film spezifische Bedeutungsschicht von `Automatismus einer Welt.

Cavell bestimmt den Film als »based upon successions of automatic world projections ${ }^{4}{ }^{48}$ Gerade deshalb aber schließt ihn Fried aus der Reihe der modernistischen Künste aus: »... the cinema, even at its most experimental, is not a modernist art. $\ll^{49}$ Cavells eigene Antwort ist komplexer: Zum einen sei auch der Film nicht völlig durch seine materielle Basis determiniert, bedeutungstragend seien vielmehr auch hier konkrete Formen wie Genres oder Figurentypen. ${ }^{50}$ Diese können ihre traditionelle Verbindlichkeit, ihren ästhetischen Automatismus verlieren und den Film so in den Modernismus stoßen. ${ }^{51}$ Zum anderen bildet die Antitheatralität des Films bei Cavell ein systematisches Komplement zur Antitheatralität der modernistischen Malerei: Handelt es sich in der modernistischen Malerei um einen Rückzug der Welt, der dialektisch die (Selbst-)Gegenwärtigkeit des Betrachtersubjekts affirmiert, "permitting us presentness to ourselves «, ${ }^{52}$ zeigt die filmische Projektion eine Welt, die keinen Betrachter zu haben scheint:

A A screen is a barrier. What does the silver screen screen? It screens me from the world it holds-that is, makes me invisible. And it screens that world from me-screens its existence from me. That the projected world does not exist (now) is the only difference from reality. . $^{53}$

Cavell artikuliert aber auch Zweifel an der Modernismusfähigkeit des Films. Sie betreffen den tiefen ethischen Ernst des Modernismus, seine »absolute condemnation to seriousness $«{ }^{54}$ einen Ernst, der sich aus der forcierten Möglichkeit des Scheiterns des jeweils in Angriff genommenen Werks ergibt. Dem Film als »Abfolge automatischer Weltprojektionen« ist diese Möglichkeit des Scheiterns aufgrund seiner besonderen technisch-medialen Struktur nicht gegeben.

\section{Komödie der Selbstreferenz}

Dem Film eignet jedoch eine spezifische Form des Komischen, wie Cavell im 16. Kapitel von The World Viewed erklärt. Mit wenigen Sätzen entwirft er dort eine Theorie der Komödie im Medium des Films. Ausgehend von Werken der Marx Brothers und Buster Keatons schreibt er: »In these instances, the comedy arises first from a transgression of mechanical or conventional conditions upon which coherent narration in film has depended. ${ }^{55}$ Und im Absatz davor heißt es: "A striking feature of these self-referential instances is that their effect is comic. $\|^{56}$ Die Andeutungen Cavells lassen sich wie folgt lesen: Das Komische im Film entsteht, wenn die filmischen Produktionsmittel im weitesten Sinne - von den technischen Apparaten bis zu den Gesten der Schauspieler - auf theatrale Weise ausgestellt werden. Der Autor spricht treffend von einer »comedy of self-reference $«{ }^{57}$ einer "Komödie der Selbstreferenz«.

Cavell handelt freilich vom Erzählfilm, von Komödien, die trotz ihres anarchischen Charakters das Ergebnis von Großproduktionen des Hollywoodkinos sind. Auch scheint es nicht ausgeschlossen, dass im Erzählfilm noch andere Formen des Komischen existieren. ${ }^{58}$ Seine Überlegungen berühren jedoch einen Grundzug in Snows experimentellem Filmschaffen: Das Komische in Snows Filmen ist das Theatralwerden der Darstellungsmittel. Mehr noch: Indem er das filmische Dispositiv theatralisiert - die technisch-mediale Basis der filmischen Antitheatralität selbst theatral macht -, rückt Snow den Film paradoxerweise ins Koordinatensystem des Modernismus. Indem er »Komödien der Selbstreferenz« inszeniert, wird der Künstler dem modernistischen Ethos radikalen Ernstes gerecht. In diesem Sinne kann auch der Titel des Films Presents verstanden werden. »Presents« meint - neben all den bereits erörterten Varianten - nicht zuletzt die aufdringliche, komische, theatrale Präsenz der filmischen Darstellungsmittel, zu denen auch die Körper der handelnden Personen zählen. Diese theatrale Präsenz aber ist zugleich ereignishaftes Geschenk, »presentness « im Sinne von Fried und Cavell. Ihr entspricht eine besondere Form ästhetischer Freiheit. Während der ethische Ernst des Modernismus in der Freiheit zu scheitern begründet ist, liegt die Freiheit von Snows Komödie der Selbstreferenz in der Möglichkeit, über Bedingungen und Bedingtheiten - sei es von Werken oder sei es der eigenen biologischen und sozialen Existenz - zu lachen.

Der zweite Akt von Presents nimmt das für die diderotsche Tradition absorptiver Bildlichkeit prägende Paradigma der vierten Wand buchstäblich. ${ }^{59}$ Die 
Kinoleinwand als eine »abschirmende Barriere«, wie Cavell sich ausdrückt, wird gegen Ende des zweiten Aktes zur Plexiglasplatte literalisiert. Diese ist transparent wie das Kameraauge, doch übt sie direkten Druck auf die im Bildfeld befindlichen Objekte aus. Sie komprimiert den Bildraum, bis die Einrichtungsgegenstände in burlesker Weise kollabieren. So macht sie den formalen Zwang, der für die Funktion des - zugleich rahmenden und mobilen - filmischen Kaschs charakteristisch ist, als Komödie sichtbar.

Die in Presents verhandelten modernistischen Parameter zeigen sich wenige Jahre zuvor in verdichteter Form in der kleinen, 15-minütigen Arbeit Breakfast (Table Top Dolly). Sie wurde 1972 auf 16-mm-Farbfilm aufgenommen und 1976 montiert. Ihr ist der letzte Abschnitt dieses Textes gewidmet. Breakfast (Table Top Dolly) radikalisiert den Topos der Bildwerdung des Raums als Operation der Verflachung. Der Film erscheint als eine ironische Anverwandlung des greenbergschen Prinzips modernistischer »flatness «. Seine visuelle Erscheinung wird durch einen einzigen vertikal in die Bildtiefe führenden Tracking Shot strukturiert, der in kleinen Schüben über einen Frühstückstisch zieht und seinem Richtungsvektor nach einer Zoombewegung vergleichbar ist. Der Tisch befindet sich direkt an einer kahlen Wand, die parallel zur Bildebene steht. Hinter ihm ist nach der Art von Fotoateliers ein dünner weißer Karton aufgehängt. Ein blau-weiß kariertes Tischtuch trägt mehr oder weniger verpackte Lebensmittel, die größtenteils in Primärfarben gehalten sind: Rot, Grün, Blau und Gelb. Man sieht Eier, einen Eierkarton, zwei transparente Plastikbecher mit Blutorangensaft, das dazugehörige Behältnis aus weißlichem Karton, (Papp-)Teller mit Süßspeisen und Fleischwaren, zwei Cocktailgläser, einen bunten Pappbecher, ein Stück Butter in Metallfolie, einen knallroten geflochtenen Korb mit einem Apfel, Trauben und einer Banane, schließlich zwei weitere Kartons mit Getränken oder Cerealien - ein Frühstücksstillleben des amerikanischen Mittelstandes.

Die Kamerafahrt hat nicht nur den optischen Effekt der Verkleinerung des Bildausschnitts und des Größerwerdens der in ihm zu sehenden Gegenstände, sondern auch unmittelbare physische Wirkungen. Je näher das Kameraauge den Gegenständen auf dem Frühstückstisch kommt, desto stärker werden sie zusammengedrückt, werden von einer unsichtbaren Kraft aus ihrer Position geschoben und an die Wand gepresst, oder sie stürzen seitlich aus dem Bildfeld. Das visuelle Bildfeld der Kamera findet sich in dieser Arbeit wie auch in Presents literalisiert, die Verdichtung des Raums vollzieht sich nicht nur optisch, sondern unmittelbar körperlich. Auch hier verwendete Snow eine vor der langsam fahrenden Kamera montierte durchsichtige Plexiglasscheibe, die wie ein Schneepflug ihre Bahn über den Frühstückstisch zieht. Am Ende des Films, während sukzessive Titel, Autor und Entstehungsjahr eingeblendet werden, fährt die Kamera mit der Plexiglasplatte dreimal rasch zurück und wieder vor in Richtung Wand und lässt den nackten Tisch entblößt und ohne Tischtuch sehen, eine für Snow typische Form der Rekapitulation und Selbstreflexion. Erst jetzt enthüllt sich die eigentliche Tiefe des Frühstückstisches, der zuvor quadratisch erschienen war, sich nun aber als längliches Objekt erweist. Zugleich wird die optische Verdichtungs- und Verkürzungsfunktion der Kameralinse deutlich.
Den ganzen Film über hört man Schab- und Raschelgeräusche aus dem Off, daneben das Klirren von Geschirr und Besteck. Einmal lässt sich eine männliche Stimme vernehmen. Im Hintergrund läuft sehr leise ein unsichtbares Radio, das an das Radio in Wavelength erinnert. Obwohl sich Ton- und Bildebene heterogen zueinander verhalten und offensichtlich nicht synchronisiert sind, neigt man dazu, die hörbaren Geräusche den im Bildfeld sichtbaren Gegenständen bzw. der mechanischen Kamerabewegung zuzuschreiben. Manchmal bilden sich aus den Geräuschen instabile rhythmische Muster, die bald wieder zerfallen. Die partielle Rhythmisierung der Tonebene sticht unmittelbar hervor, wenn sie eintritt, und fungiert so als ein zugleich formaler und theatraler Effekt. Es handelt sich genau genommen um eine Theatralität der akustischen Form selbst.

Das Dispositiv von Breakfast (Table Top Dolly) drückt einerseits den konstitutiven Faktor von Gewalt aus, der jeglicher Bildwerdung als Kadrierung innewohnt, andererseits aber einen erstaunlichen Aspekt anarchischer Freiheit. Die Konsumgüter auf dem Frühstückstisch verlieren ihren angestammten Zweck der biologischen und symbolischen Reproduktion der kleinbürgerlichen Familie der westlichen Welt der 1970er Jahre und führen eine kleine Komödie der Dinge auf. Sie gewinnen eine unbeholfene und witzige Physiognomik, die sie beinahe als Lebewesen erscheinen lässt. Wenn sie durch Zufall flach auf der bildparallelen Glasplatte zu liegen kommen, erscheinen sie hingegen wie abstrakte geometrische Formen. So spielen in Breakfast (Table Top Dolly) die Parameter von Formalismus und Theatralität, von Programm und Freiheit, von Konstruktion und Improvisation, von Gewalt und anarchischer Komödie zusammen.

Der Film lässt sich aber auch als ironischer Kommentar auf die Dialektik von literal shape - als Format bzw. physische Form des Bildträgers - und depicted shape - als Gefüge der das Bildfeld strukturierenden Binnenformen - verstehen, eine Dialektik, die Fried zufolge den grundlegenden formalen Impetus der avanciertesten Malerei der 1960er Jahre ausmachte - bei Kenneth Noland, Jules Olitski und nicht zuletzt in Stellas Serie der Irregular Polygons (1965/66). ${ }^{60}$ Breakfast (Table Top Dolly) setzt die zwingende Macht der literal shape des filmischen Kaschs über die depicted shape der Alltagsgegenstände des Bildfeldes in Szene, die gerade als projizierte Formen die Körperlichkeit ihrer Referenzobjekte anzunehmen scheinen. Zugleich wird die Form des Kaschs, seine literal shape, nicht nur in der Funktion einer rechteckigen lateralen Begrenzung erfahrbar, vielmehr zeigt sich seine Grenze nach svorne`, zur Kamera bzw. zum Publikum hin, als die entscheidende. Sie demarkiert die Zone jenes absoluten Offs, welches im Gegensatz zu den Bereichen des relativen Offs jenseits der seitlichen Kanten des Bildfeldes niemals in den Blick der aufnehmenden bzw. projizierenden Instanz rücken kann. Die Kamerabewegung, welche das absolute Off im Kasch des Bildfeldes zur Geltung bringt, ist der Zoom bzw. die Kamerafahrt vertikal in die Bildtiefe, das strukturierende Programm von Snows kleinem Film. Doch nicht nur dieser, alle der hier diskutierten Arbeiten Snows machen das absolute Off im projizierten Filmbild selbst vernehmbar, und sie besetzen es doppelt - als Sphäre der Produktion und der Rezeption zugleich. 
Für die Produktionssphäre ist die Hybridisierung von körperlichen Gesten und technischen Operationen bestimmend, die eine ungelenke Freiheit zum Ausdruck bringt. Die Rezeptionssphäre hingegen überblendet das filmische Dispositiv mit dem Dispositiv des Theaters - eines Theaters freilich, das nicht dem diderotschen Modell von Tableau und Absorption folgt, sondern der Theatralität und dem Spektakel verpflichtet ist. Dieser Befund mag befremdlich anmuten. Denn Fried und Cavell betonen ja, dass der Film als fotografisches Medium die Grenze zwischen der in seinen Bildern erscheinenden, strukturell vergangenen Welt und der Welt des Publikums verabsolutiere, und zwar nicht als Ergebnis einer historischen Reihe künstlerischer Errungenschaften, sondern gewissermaßen von selbst, als Folge der spezifischen Beschaffenheit der ihn hervorbringenden Apparate. Der Widerspruch ist jedoch nur ein scheinbarer. Denn der technische Automatismus des Mediums vermag niemals die Notwendigkeit eines bestimmten Automatismus im Ästhetischen zu begründen.

Wie geht Breakfast (Table Top Dolly) mit dem Gegensatz von technischem und ästhetischem Automatismus um? Zum einen überaffirmiert die Arbeit das Paradigma des Films als eines technisch-medial bedingten absorptiven Mediums. Die Grenze zwischen der Welt des Films und der Welt des Publikums wird als durchsichtige Plexiglasplatte vergegenständlicht, die das Linsensystem der Kamera in seiner physischen Dimension und nicht nur in seinen rein optischen - man möchte sagen ideologischen $<$ - Wirkungen erfahrbar werden lässt. Zum anderen hält sich der Künstler nicht an die dem filmischen Medium inhärente Vorentscheidung der Absorption, er konterkariert sie vielmehr durch die theatralischen 'Schicksale $<$ - die Lage- und Formveränderungen - der Gegenstände auf dem Frühstückstisch, die direkt an das Lachen des Publikums appellieren.

Dennoch macht Breakfast (Table Top Dolly) eine nach Cavell grundlegende mediale Eigenschaft des filmischen Kaschs thematisch - "Gussform« oder einfach nur emphatisch »Form « zu sein: »The screen is a frame; the frame is the whole field of the screen - as a frame of film is the whole field of a photograph, like the frame of a loom or a house. In this sense, the screen-frame is a mold, or form. ${ }^{61}$

Wenn Cavell an dieser Stelle von »Form« spricht, meint er nicht bloß den konkreten Umriss des Kaschs, sondern das Zusammenwirken zwischen dem Format des Kaschs und dem, was er zu sehen gibt. Diese - emphatische - Form des kinematographischen Bildfeldes ist zum einen durch Ausschnitthaftigkeit und Beweglichkeit charakterisiert. Im Rahmen des filmischen Kaschs kann scheinbar die gesamte Welt sukzessive Bild werden. Zum anderen aber eignet dem filmischen Medium - hier ist freilich vom Zelluloidfilm die Rede - ein Zwang, ein Automatismus technischer Natur, der die Filmbilder mittels eines fotografischen Abbildungsmechanismus erzeugt, ohne das Spiel kleinster Handlungen und Intentionen, welches den malerischen Akt kennzeichnet. Die rechteckige literal shape des Kaschs lässt sich als unmittelbarer Ausdruck des technischen Automatismus des Films verstehen, ebenso das elementare Repertoire der Kamera- und Zoombewegungen, das Snow in seinen Filmen untersucht. Während das Verhältnis von literal shape und depicted shape nach Fried in der avancierten modernistischen Malerei der 1960er Jahre stets aufs Neue verhandelt werden kann, ja verhandelt werden muss, sodass bei Stella schließlich die Form des Bildträgers selbst zur Disposition gestellt wird, sind die Struktur des filmischen Kaschs und die Operationen seiner Verschiebung technisch bedingt.

Wie Presents leugnet Breakfast (Table Top Dolly) die formatierende Gewalt des Kaschs über die im Bildfeld befindlichen Objekte nicht. Die Arbeit lässt sie vielmehr als solche erscheinen, »erkennt sie an«, um mit Fried und Cavell zu sprechen. Darin liegen ihre selbstreflexive Dimension und ihr Modernismus. Der Modus dieses Erscheinens aber ist nicht der nackte modernistische Ernst, sondern die Komödie. Denn die Wirkungen des Kaschs auf die Formen der Frühstückswaren im Bildfeld sind katachrestisch gewendet. Es handelt sich nicht nur um rein visuelle, sondern auch um buchstäblich physische Wirkungen. Breakfast (Table Top Dolly) verleiht dem Kasch eine theatrale und gestische Dimension, als wäre es nicht der technische Automatismus eines Mediums, der die Transformationen und Ortsverschiebungen der Waren auf dem Frühstückstisch bewirkt, sondern ein obsessiver kinematographischer Wille, der die Relation von literal shape und depicted shape zum Gegenstand seiner linkischen Forschungen macht. 


\section{Endnoten}

Hier und im Folgenden wird nicht streng zwischen skinematographisch und filmisch bzw. zwischen ,Kino und Film < unterschieden. Für Snow selbst besteht jedoch ein solcher Unterschied. Seine explizit für die Projektion im Kinosaal konzipierten Arbeiten nennt er »kinematographisch«. Sie sind dadurch gekennzeichnet, dass ihre gesamte Dauer werkkonstitutiv ist, dass man sie also von Anfang bis Ende sehen muss, um einen adäquaten ästhetischen Eindruck zu erlangen. Vgl. Michael Snow, Moving Images - Scene One: Cinema (Film), in: Gloria Moure (Hg.), Michael Snow. Sequences. A History of His Art, Barcelona 2015, S. 123-155, hier S. 123. In diesem Sinne handelt es sich bei allen im vorliegenden Text untersuchten Filmen Snows um kinematographische Werke.

2 Michael Snow, Statements/18 Canadian Artists [1967], in: ders., The Collected Writings of Michael Snow, Waterloo (Ontario) 1993, S. 26-27, hier S. 26.

3 Michael Fried, Art and Objecthood [1967], in: ders., Art and Objecthood. Essays and Meviews, Chicato and Objecthood [1967], in: ders., A A

Reviews, Chicago/London 1998, S. 148-172, hier S. 164.

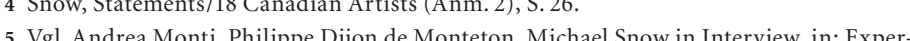
imental Conversations 3, Frühjahr 2009, unter: http://experimentalconversations.com/ article/michael-snow-in-interview [07.01. 2018].

6 Vgl. Diedrich Diederichsen, Case Study New York um 1960. Sex und Gewalt statt Lust und Unlust, in: ders., Körpertreffer. Zur Ästhetik der nachpopulären Künste, Berlin 2017, S. 50-97; ders., Das Primäre. Minimal Music, Minimal Art, Maximal Pop, in: Andreas Meyer (Hg.), Was bleibt? 100 Jahre Neue Musik, Mainz 2011, S. 162-177.

7 Ein vergleichbarer Zusammenhang lässt sich für gewisse Spielarten der Konzeptkunst behaupten. Sol LeWitt wäre ein Beispiel.

8 Vgl. Stanley Cavell, The World Viewed. Reflections on the Ontology of Film, Cambridge (Mass.)/London 1979, S. 101-108.

9 Ebd., S. 104

10 Ebd., S. 113 .

11 Vgl. Immanuel Kant, Kritik der praktischen Vernunft, hg. v. Horst D. Brandt und Heiner F. Klemme, Hamburg 2003, S. 44f [Akademieausgabe, S. 33], S. 148 [AA, S. 109]: ders Grundlegung zur Metaphysik der Sitten, in ders, Gesammelte Schriften, ho v. Königlich PreßBischen Akademie der Wissenschaften, Bd. 4 Belin 1911, S. 385-463, hier S. 406-445.

12 Vgl. Kant, Grundlegung zur Metaphysik der Sitten (Anm. 11), S. 452.

13 Snow, Moving Images - Scene One (Anm. 1), S. 148.

14 Vgl. ebd., S. 128. Der Terminus ,formal events stammt nicht von Snow selbst, sondern vom Autor des vorliegenden Textes.

15 Eine besondere Pointe von New York Eye and Ear Controlbesteht darin, der tableauhaften Kadrierung der Skulptur eine weitere Ebene der Kadrierung hinzuzufügen, jene des mobilen filmischen Kasch

16 Vgl. David Thomas, Vertov, Snow, Farocki. Machine Vision and the Posthuman, New York/London 2013, S. S. 101-185; vgl. auch Annette Michelson, About Snow, in: October 8, Frühjahr 1989, S. 111-125, hier S. 119-123.

17 Zur Kardinalitä̈t des menschlichen Körpers, das heißt zu den von seiner organischen und perzeptiven Struktur vorgegebenen Koordinaten, vgl. Meyer Schapiro, On Some Problems in the Semiotics of Visual Art, Field and Vehicle in Image-Signs, in: Simiolus. Netherlands Quarterly for the History of Art 6/1, 1972/73, S. 9-19; vgl. auch David Summers, Real Spaces, London/New York 2003, S. 36-41, 361-395.

18 Vgl. Gilles Deleuze, Kino 1. Das Bewegungs-Bild, Frankfurt a. M. 2005, S. 155-170.

19 Zum Problem eines posthumanen maschinellen Sehens im Werk von Snow vgl. Thomas, Vertov, Snow, Farocki (Anm. 16), S. 75-216.

20 Vgl. ebd., S. 147-155; zum konkreten Aufbau der für die Herstellung von La Région Centrale verwender verwend win Con
21 Vgl. Michael Fried, Why Photography Matters as Art as Never Before, New Haven/London 2008, S. 26

22 Zum historischen und systematischen Verhältnis von Absorption und Theatralität vgl. Michael Fried, Absorption and Theatricality. Painting and Beholder in the Age of Diderot, Chicago/London 1980; ders., Between Realisms. From Derrida to Manet, in: Critical Inquiry 21/1, Herbst 1994, S. 1-36, hier S. 4-7.

23 Vgl. Bart Testa, An Axiomatic Cinema. Michael Snow's Films, in: Jim Shedden (Hg.), Presence and Absence. The Films of Michael Snow. 1956-1991, Toronto 1995, S. 27-83, hier S. 52; für die Goya-Referenz vgl. das folgende Interview: Ionathan Rosenbaum, The "Presents" of Michael Snow, in: Film Comment 3/17, Mai/Juni 1981, S. 35-38, unter: https://jonathanrosenbaum net/1981/05/the-presents-of-michael-snow [28 01 2018] Zum Slapstick bei Snow in Relation zu den Filmen Buster Keatons vgl. Eric Bullot, Keaton Derbst 2005, S. 17-28.

25 Die Filmbilder des dritten Aktes sind absorptiv, weil sie Blicke ohne Blickträger darstellen. Sie sind zugleich theatral, weil sie Hunderte Male wieder und wieder eine grundlegende Form der Kamerabewegung exemplifizieren - den Schwenk.

26 Zur Geschichte einer Ästhetik der Schwere und ihrer Verflechtung mit dem Paradigma ästhetischer Einfühlung vgl. Malika Maskarinec, The Forces of Form in German Modernism, Evanston (Ill.) 2018.

27 Peter Szondi, Tableau und coup de thêâtre. Zur Sozialpsychologie des bürgerlichen Trauerspiels bei Diderot. Mit einem Exkurs über Lessing, in: ders., Schriften, Bd. 2, Frankfurt a. M. 1978, S. 205-232, hier S. 212.

28 Ebd.

29 Ebd., S. 224; vgl. auch ebd., S. 231f.

30 Vgl. ebd., S. 219f.; zur Vorgeschichte des "genre sérieux« bei Pierre Corneille vgl. Peter Szondi, Die Theorie des bürgerlichen Trauerspiels im 18. Jahrhundert. Studienausgabe der Vorlesungen, Bd. 1, hg. v. Gert Mattenklott, Frankfurt a. M. 2006, S. 91-100.

31 Die Diderot-Bezüge in Snows CEuvre sind vielfältig. Leider muss eine Analyse von Snows monumentalem Film Rameau's Nephew by Diderot (Thanx to Dennis Young) by Wilma Schoen, der Diderot ja schon im Titel trägt, im Rahmen des vorliegenden Aufsatzes unterbleiben und der Zukunft vorbehalten werden.

$32 \mathrm{Vgl}$. Snow, Moving Images - Scene One (Anm. 1), S. 144

32 Vgl. Snow,
33 Vgl. ebd.

34 In diesem Zusammenhang ist auf eine weitere, von Snow ins Treffen geführte Bedeutuns

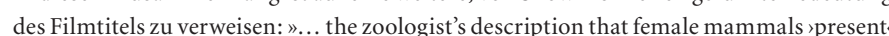
to males is pertinent to the film Presents, « Snow, Moving Images - Scene One (Anm. 1) S. 143.

35 Vgl. Martin Heidegger, Die Zeit des Weltbildes [1938], in: ders., Gesamtausgabe, 1. Abt. Bd. 5: Holzwege, Frankfurt a. M. 1977, S. 75-113.

36 Cavell, The World Viewed (Anm. 8), S. 11

37 Dem Optimismus Cavells könnte der Pessimismus Niklas Luhmanns gegenübergestell werden, der - ebenfalls aus einer der hegelschen Philosophie verpflichteten Perspektive über das ästhetische Programm der deutschen Romantik schreibt: „Dieser Ausweg endet in der Tragik des sich selbst beobachtenden Individuums, das die Ergebnislosigkeit dieser Bemühung, das Sich-nicht-Bestimmenkönnen, als Kunstwerk zelebriert. Der Eindruck ist dann: daß das Individuum sich für die eigene Intransparenz, für die Selbstintransparenz, dadurch entschädigt, daß es sich als Kunstwerk transparent macht." Niklas Luhmann, Weltkunst, in: ders., Schriften zur Kunst und Literatur, hg. v. Niels Weber, Frankfurt a. M. 2008, S. 189-245, hier S. 189.

38 Cavell, The World Viewed (Anm. 8), S. 127; vgl. auch ebd., S. 110, 117

39 Vgl. ebd., S. 104, 114

40 "A modernist art, investigating its own physical basis, searching out its own conditions of existence, rediscovers the fact that its existence as an art is not physically assured." Ebd., S. 107. 


\section{Endnoten}

41 Ebendiesen Befund versucht Fried am Beispiel Stellas zu belegen. Vgl. Michael Fried, Shape as Form. Frank Stella's Irregular Polygons, in: ders., Art and Objecthood. Essays and Reviews, Chicago/London 1998, S. 77-99.

42 Vgl. Stanley Cavell, Music Discomposed, in: ders., Must We Mean What We Say? A Book of Essays, Cambridge 1998, S. 180-212.

43 Vgl. Cavell, The World Viewed (Anm. 8), S. 110f.

44 Ebd., S. 117

45 Vgl. ebd., S. 22f. Diese Ưberlegungen gehören zu den dunkelsten des Buches und können

hier nicht ausführlich diskutiert werden. Cavell scheint jedoch so etwas wie eine Selbsterkenntnis des modernen Subjekts in seinen historischen und epistemologischen Bedingtheiten und Bedingungen zu meinen.

46 Vgl. Fried, Art and Objecthood (Anm. 3), S. 164, 171: vgl.ders, Why Photography

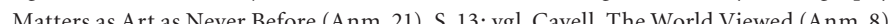
S. 103 , 118.0 S.

Cavell, The World Viewed (Anm. 8), S. 23

Vgl. ebd, S. 72f., 118.

Fried, Art and Objecthood (Anm. 3), S. 164

50 Vgl. Cavell, The World Viewed (Anm. 8), S. 105

51 Vgl. ebd., S. 68-73, 103.

52 Ebd., S. 23.

53 Ebd., S. 24.

54 Ebd., S. 118.

55 Ebd., S. 126.

56 Ebd., S. 125

57 Ebd., S. 126.

58 Für einen alternativen Zugang zu Keatons Filmkomödien vgl. etwa Sulgi Lie, Quasi-Körper. Fiktion, Infrafiktion und Metafiktion in der Filmkomödie, in: Thomas Hilgers, Gertrud Koch (Hg.), Fiktion und Perspektivität, München 2017, S. 179-194.

59 Hier wäre an die weitverbreitete Funktion von Katachresen als Vehikel des Komischen zu erinnern.

$60 \mathrm{Vgl}$. Fried, Shape as Form (Anm. 41).

61 Cavell, The Woild Viewed (Ang, B), S.25. 